\title{
A Multiple Integral Evaluation Inspired by the Multi-WZ Method*
}

\author{
Akalu Tefera \\ Department of Mathematics \\ Temple University, Philadelphia, PA 19122. \\ akalu@math.temple.edu
}

\begin{abstract}
We give an integral identity which was conjectured and proved by using the continuous version of the multi-WZ method.
\end{abstract}

Submitted: May 25, 1999; Accepted: October 20, 1999.

Mathematical Reviews Subject Numbers: 05, 33.

\section{Introduction}

There are relatively few known non-trivial evaluations of $k$-dimensional integrals, with arbitrary $k$. Celebrated examples are the Selberg and the Mehta-Dyson integrals, as well as the Macdonald constant term ex-conjectures for the various root systems. They are all very important. See [AAR98] for a superb exposition of the various known proofs and of numerous intriguing applications.

At present, the (continuous version of the) WZ method [WZ92] is capable of mechanically proving these identities only for a fixed $k$. In principle for any fixed $k$ (even, say, $k=100000$ ), but in practice only for $k \leq 5$. However, by interfacing a human

*This work will appear in the author's Ph.D. thesis. 
to the computer-generated output, the human may discern a pattern, and generalize the computer-generated proofs for $k=1,2,3,4$ to an arbitrary $k$.

Using this strategy, Wilf and Zeilberger [WZ92] gave a WZ-style proof of Selberg's integral evaluation. In this article we present a new multi-integral evaluation, that was first found by using the author's implementation of the continuous multi-WZ method which is called SMint ${ }^{1}$. Both the conjecturing part, and the proving part, were done by a close human-machine collaboration. Our proof hence may be termed computer-assisted but not yet computer-generated.

Now that the result is known and proved, it may be of interest to have a non-WZ proof, possibly by performing an appropriate change of variables, converting the multiintegral to a double integral. My advisor, Doron Zeilberger, is offering $\$ 100$ for such a proof, provided it does not exceed the length of the present proof.

Since a key to the integral evaluation is the package SMint, first we give a brief description of the package.

\section{A Brief Description of SMint}

The "objects" of study in the continuous version of the multi-WZ theory are expressions of the kind

$$
\int F(n, \mathbf{m}, \mathbf{y}, \mathbf{x}) d \mathbf{x}
$$

and identities between them. In the above general integral-sum, $n$ is a discrete variable, $\mathbf{m}$ is a discrete multi-variable, while $x$ and $y$ are continuous multi-variables, and $F$ is hypergeometric in all its arguments.

For a given hypergeometric function $F(n, \mathbf{m}, \mathbf{y}, \mathbf{x})$, where $\mathbf{y}=\left(y_{1}, \ldots, y_{k}\right)$, we look for a recurrence operator $\sum_{i=0}^{I} a_{i}(n) E_{n}^{i}$, where $a_{i}(n)$ polynomial in $n$ and $E_{n}$ is the forward shift operator in $n$, and a $k$-tuple of rational functions(the certificate) $\left[R_{1}, \ldots, R_{k}\right]$ $\left(R_{i}=R_{i}(n, \mathbf{m}, \mathbf{y}, \mathbf{x})\right)$ such that the recurrence-differential operator

$$
\sum_{i=0}^{I} a_{i}(n) E_{n}^{i}-\sum_{j=1}^{k} D_{x_{j}} \cdot R_{j}
$$

\footnotetext{
${ }^{1}$ available from http: //www. math.temple.edu/ akalu/maplepack/SMint
} 
annihilates $F$,i.e.,

$$
\sum_{i=0}^{I} a_{i}(n) F(n+i, \mathbf{m}, \mathbf{y}, \mathbf{x})-\sum_{j=1}^{k} D_{x_{j}}\left(R_{j} F\right)=0 .
$$

The existence of an operator of the above form is guaranteed by the fundamental theorem of the (continuous version of the) multi-WZ theory [WZ92].

Doron Zeilberger wrote a Maple implementation, TRIPLE_INTEGRAL ${ }^{2}$, that performs the algorithm described in [WZ92] for the case of three continuous variables $(k=3)$. But TRIPLE_INTEGRAL does not completely automate the method, for instance, it requires the user to guess the denominators of the $R_{i}$ 's.

The author wrote two Maple packages Mint and SMint which improved and generalized Zeilberger's TRIPLE_INTEGRAL for any specific number of continuous variables so that it completely automates the continuous multi-WZ method. The package SMint is specially designed to handle identities which invlove pure multiple integrals where the integrand is symmetric w.r.t. the integration variables. The detailed technical description of Mint and SMint is available from the author's home page ${ }^{3}$ and will also appear in a forthcoming paper [T99].

\section{Notation}

In the sequel, $k$ is a positive integer, $m$ and $n$ are non-negative integers. The notations used in this article are defined as follows.

$$
\begin{aligned}
\mathbf{x} & :=\left(x_{1}, \ldots, x_{k}\right), \\
\hat{\mathbf{x}}_{i} & := \begin{cases}\left(x_{2}, \ldots, x_{k}\right) \\
\left(x_{1}, \ldots, x_{i-1}, x_{i+1}, \ldots, x_{k}\right) & \text { for } i=1 \\
\left(x_{1}, \ldots, x_{k-1}\right) & \text { for } i=k\end{cases} \\
d \mathbf{x} & =d x_{1} \cdots d x_{k}, \\
(y)_{m} & := \begin{cases}1 & \text { for } m=0 \\
\prod_{i=0}^{m-1}(y+i) & \text { for } m>0\end{cases} \\
e_{1}(\mathbf{x}) & :=\sum_{i=1}^{k} x_{i}, \\
e_{2}(\mathbf{x}) & :=\sum_{1 \leq i<j \leq k} x_{i} x_{j},
\end{aligned}
$$

\footnotetext{
2 available from http://www.math.temple.edu/ zeilberg/

${ }^{3}$ http://www . math.temple.edu/ akalu/
} 


$$
\begin{aligned}
\Delta_{n} F(n, \mathbf{x}) & :=F(n+1, \mathbf{x})-F(n, \mathbf{x}), \\
D_{x} & :=\frac{\partial}{\partial x}
\end{aligned}
$$

\section{The Integral Evaluation}

\section{Theorem}

$\int_{[0,+\infty)^{k}}\left(e_{2}(\mathbf{x})\right)^{m}\left(e_{1}(\mathbf{x})\right)^{n} e^{-e_{1}(\mathbf{x})} d \mathbf{x}=\frac{m !(2 m+n+k-1) !(k / 2)_{m}}{(2 m+k-1) !}\left(\frac{2(k-1)}{k}\right)^{m} T_{k}(m)$

for any positive integer $k$, and for all non-negative integers $m$ and $n$, where,

$$
T_{k}(m)-T_{k}(m-1)=\frac{(k(k-2))^{m}((k-1) / 2)_{m}}{(k-1)^{2 m}(k / 2)_{m}} T_{k-1}(m) \quad k \geq 2,
$$

$T_{1}(m)=0, m \geq 0$, and $T_{k}(0)=1, k \geq 2$.

\section{Proof of the Integral Evaluation}

If $k=1$, then trivially, both sides of the integral equate to zero. Let $k>1$ and $A_{k}(m, n)$ be the left side of the integral divided by

$$
\frac{m !(2 m+n+k-1) !(k / 2)_{m}}{(2 m+k-1) !}\left(\frac{2(k-1)}{k}\right)^{m}
$$

We want to show $A_{k}(m, n)=T_{k}(m)$, for all $m, n$ in $\mathbb{Z}_{\geq 0}$. Let

$$
F_{k}(m, n ; \mathbf{x}):=\frac{(2 m+k-1) !}{m !(2 m+n+k-1) !(k / 2)_{m}}\left(\frac{k}{2(k-1)}\right)^{m}\left(e_{2}(\mathbf{x})\right)^{m}\left(e_{1}(\mathbf{x})\right)^{n} e^{-e_{1}(\mathbf{x})}
$$

We construct ${ }^{4}$

$$
R\left(u ; v_{1}, \ldots, v_{k-1}\right):=\frac{u}{2 m+n+k}
$$

with the motive that

$$
\Delta_{n} F_{k}(m, n ; \mathbf{x})=-\sum_{i=1}^{k} D_{x_{i}}\left[R\left(x_{i} ; \hat{\mathbf{x}}_{i}\right) F_{k}(m, n ; \mathbf{x})\right] .
$$

\footnotetext{
${ }^{4}$ The production of the rational function $R$ and the corresponding recurrence-differential equation was done automatically by SMint for $k=2,3,4$, and the output is available from http://www . math. temple.edu/ akalu/maplepack/rational1. output
} 
Now, we verify (WZ 1),

$$
\begin{aligned}
\frac{F_{k}(m, n+1 ; \mathbf{x})-F_{k}(m, n ; \mathbf{x})+\sum_{i=1}^{k} D_{x_{i}}\left[R\left(x_{i} ; \hat{\mathbf{x}}_{i}\right) F_{k}(m, n ; \mathbf{x})\right]}{F_{k}(m, n ; \mathbf{x})} \\
=\frac{F_{k}(m, n+1 ; \mathbf{x})}{F_{k}(m, n ; \mathbf{x})}-1+\sum_{i=1}^{k} D_{x_{i}}\left[R\left(x_{i} ; \hat{\mathbf{x}}_{i}\right)\right]+R\left(x_{i} ; \hat{\mathbf{x}}_{i}\right) D_{x_{i}}\left[\log \left(F_{k}(m, n ; \mathbf{x})\right)\right] \\
=\frac{e_{1}(\mathbf{x})}{2 m+n+k}-1+\frac{k}{2 m+n+k}+ \\
\quad \sum_{i=1}^{k}\left(\frac{n}{e_{1}(\mathbf{x})} \frac{x_{i}}{2 m+n+k}+\frac{m e_{1}\left(\hat{\mathbf{x}}_{i}\right)}{e_{2}(\mathbf{x})} \frac{x_{i}}{2 m+n+k}-\frac{x_{i}}{2 m+n+k}\right) \\
=\frac{e_{1}(\mathbf{x})}{2 m+n+k}-1+\frac{k}{2 m+n+k}+\frac{n}{2 m+n+k}+\frac{2 m}{2 m+n+k}-\frac{e_{1}(\mathbf{x})}{2 m+n+k} \\
=0 .
\end{aligned}
$$

Hence, by integrating both sides of (WZ 1) w.r.t. $x_{1}, \ldots, x_{k}$ over $[0, \infty)^{k}$, we get

$$
A_{k}(m, n+1)-A_{k}(m, n) \equiv 0 .
$$

To complete the proof we show $A_{k}(m, 0)=T_{k}(m)$ for all $m$ in $\mathbb{Z}_{\geq 0}$.

To this end, set $A_{k}(m):=A_{k}(m, 0)$ and $F_{k}(m ; x):=F_{k}(m, 0 ; \mathbf{x})$. Now, we construct ${ }^{5}$,

$$
R\left(u ; v_{1}, \ldots, v_{k-1}\right):=\frac{\left((k-1)(m+1)+e_{1}\left(v_{1}, \ldots, v_{k-1}\right)\right) u+e_{2}\left(v_{1}, \ldots, v_{k-1}\right)}{(k-1)(m+1)(2 m+k)}
$$

with the motive that

$$
F_{k}(m+1 ; \mathbf{x})-F_{k}(m ; \mathbf{x})=-\sum_{i=1}^{k} D_{x_{i}}\left[R\left(x_{i} ; \hat{\mathbf{x}}_{i}\right) F_{k}(m ; \mathbf{x})\right] .
$$

Verification of (WZ 2):

$$
\begin{aligned}
& \frac{F_{k}(m+1 ; \mathbf{x})-F_{k}(m ; \mathbf{x})+\sum_{i=1}^{k} D_{x_{i}}\left[R\left(x_{i} ; \hat{\mathbf{x}}_{i}\right) F_{k}(m ; \mathbf{x})\right]}{F_{k}(m ; \mathbf{x})} \\
& =\frac{F_{k}(m+1 ; \mathbf{x})}{F_{k}(m ; \mathbf{x})}-1+\sum_{i=1}^{k} D_{x_{i}}\left[R\left(x_{i} ; \hat{\mathbf{x}}_{i}\right)\right]+\sum_{i=1}^{k} R\left(x_{i} ; \hat{\mathbf{x}}_{i}\right) D_{x_{i}}\left[\log \left(F_{k}(m ; \mathbf{x})\right)\right] \\
& =\frac{k e_{2}(\mathbf{x})}{(m+1)(k-1)(2 m+k)}-1+\sum_{i=1}^{k} \frac{(k-1)(m+1)+e_{1}\left(\hat{\mathbf{x}}_{i}\right)}{(m+1)(k-1)(2 m+k)}+
\end{aligned}
$$

\footnotetext{
${ }^{5}$ The production of the rational function $R$ and the corresponding recurrence-differential equation was done automatically by SMint for $k=2,3,4,5$ and the output is available from http://www. math. temple. edu/ akalu/maplepack/rational2. output
} 


$$
\begin{aligned}
& \sum_{i=1}^{k} \frac{\left.(k-1)(m+1) x_{i}+e_{2}(\mathbf{x})\right)}{(m+1)(k-1)(2 m+k)}\left(\frac{m e_{1}\left(\hat{\mathbf{x}}_{i}\right)}{e_{2}(\mathbf{x})}-1\right) \\
= & \frac{k e_{2}(\mathbf{x})}{(m+1)(k-1)(2 m+k)}-1+\frac{k}{2 m+k}+\frac{e_{1}(\mathbf{x})}{(m+1)(2 m+k)}+\frac{2 m}{2 m+k} \\
& -\frac{e_{1}(\mathbf{x})}{2 m+k}+\frac{m e_{1}(\mathbf{x})}{(m+1)(2 m+k)}-\frac{k e_{2}(\mathbf{x})}{(m+1)(k-1)(2 m+k)} \\
= & 0 .
\end{aligned}
$$

Hence, by integrating both sides of (WZ 2) w.r.t. $x_{1}, \ldots, x_{k}$ over $[0, \infty)^{k}$, we obtain,

$$
A_{k}(m+1)-A_{k}(m)=\frac{(k(k-2))^{m+1}((k-1) / 2)_{m+1}}{(k-1)^{2(m+1)}(k / 2)_{m+1}} A_{k-1}(m+1),
$$

and noting that $A_{k}(0)=1, k \geq 2, A_{1}(m)=0$, it follows that $A_{k}(m)=T_{k}(m)$, for all $m$ in $\mathbb{Z}_{\geq 0}$. Consequently, $A_{k}(m, n)=T_{k}(m)$ for all $m, n$ in $\mathbb{Z}_{\geq 0}$.

By unfolding the recurrence equation for $T_{k}(m)$, we obtain the following identity.

\section{Corollary}

$$
\begin{gathered}
\int_{[0,+\infty)^{k}}\left(e_{2}(\mathbf{x})\right)^{m}\left(e_{1}(\mathbf{x})\right)^{n} e^{-e_{1}(\mathbf{x})} d \mathbf{x}=\frac{m !(2 m+n+k-1) !(k / 2)_{m}}{(2 m+k-1) !}\left(\frac{2(k-1)}{k}\right)^{m} \\
\left(1+\sum_{r=1}^{k-2} \sum_{1 \leq s_{r} \leq \cdots \leq s_{1} \leq m} \prod_{i=1}^{r} \frac{\left((k-i)^{2}-1\right)^{s_{i}}((k-i) / 2)_{s_{i}}}{(k-i)^{2 s_{i}}((k-i+1) / 2)_{s_{i}}}\right)
\end{gathered}
$$

\section{Remarks}

1. From the computational point of view, the recurrence form of the integral is nicer than its indefinite summation form (the above corollary), for the former requires $O(m k)$ calculations, whereas the latter requires $O\left(m^{k}\right)$ calculations. However, in both forms the evaluation of the right side of the integral is much faster (for specific $m, n$, and $k$ ) than the direct evaluation of the left side of our intergal. Hence both forms are indeed complete answers in the sense of Wilf [W82].

2. The present paper is an example of what Doron Zeilberger [Z98] calls WZ Theory, Chapter 1 1/2. Even though, at present, our proof, for general $k$, was human-generated, it seems that by using John Stembridge's [S95] Maple package for symmetric functions, $S F$, or an extension of it, it should be possible to write a new version of SMint that should work for symbolic, i.e. arbitrary, $k$, thereby fulfilling the hope raised in [Z98]. 
THE ELECTRONiC JOURNAL OF COMBINATORICS 6(1999), \#N2

Acknowledgement: I thank Doron Zeilberger, my Ph.D. thesis advisor, for very helpful suggestions and valuable support.

\section{References}

[AAR98] G. Andrews, R. Askey, and R. Roy, Special Functions, Cambridge University Press, 1998.

[S95] J.R. Stembridge, A Maple package for symmetric functions, J. Symbolic Comput., 20(1995), 755-768.

[T99] A. Tefera, Complete Automation of the Continuous Multi-WZ Method, in preparation.

[W82] H.S. Wilf, What is an answer?, Amer. Math. Monthly, 89 (1982), 289-292.

[WZ92] H.S. Wilf and D. Zeilberger, An Algorithmic proof theory for hypergeometric (ordinary and "q") multisum/integral identities, Invent. Math., 108 (1992), 575-633.

[Z98] D. Zeilberger, WZ Theory, Chapter II, The Personal Journal of S.B. Ekhad and D. Zeilberger, http://www.math.temple.edu/ zeilberg/pj.html. 